\title{
NOTE
}

\section{SUPERVIVENCIA DE VIEIRA PATAGÓNICA DE TALLA NO COMERCIAL DESCARTADA POR LA PESQUERÍA}

\author{
Matías Schwartz ${ }^{1}$, Mariana Escolar ${ }^{1}$, Ángel Marecos $^{1,2}$, Susana Herrera ${ }^{1}$, \\ Ronaldo Diaz ${ }^{1}$, JaVier Villalba ${ }^{1}$ y Silvana CAMPodónico ${ }^{1}$ \\ ${ }^{1}$ Instituto Nacional de Investigación y Desarrollo Pesquero (INIDEP), Paseo Victoria Ocampo $\mathrm{N}^{\mathrm{o}} 1$, \\ Escollera Norte, B7602HSA - Mar del Plata, Argentina \\ correo electrónico: schwartz@inidep.edu.ar \\ ${ }^{2}$ Consejo Nacional de Investigaciones Científicas y Técnicas (CONICET), Argentina
}

\begin{abstract}
RESUMEN. La pesquería bentónica más importante en extensión y producción del Atlántico Sudoccidental es la de vieira patagónica (Zygochlamys patagonica). La desarrollan cuatro buques factoría con redes de arrastre de fondo no selectivas y planta procesadora a bordo. Durante el proceso de selección se retienen las vieiras de talla comercial $(\geq 55$ $\mathrm{mm}$ de alto total) y las que no la alcanzan $(<55 \mathrm{~mm} \mathrm{AT})$ se descartan al mar. El objetivo del presente trabajo fue determinar el nivel de supervivencia de los ejemplares descartados al mar y el efecto del proceso de selección a bordo. Se seleccionaron vieiras de talla no comercial procedentes de cubierta (control) y descarte (tratamiento). Se las clasificó de acuerdo con el nivel de daño y se las colocó en bolsas de polipropileno para fondearlas ( $\sim 100 \mathrm{~m}$ de profundidad). Cuando se recuperaron los fondeos, a los 10 y 54 d, se contabilizó la cantidad de individuos vivos en cada bolsa. Los mayores niveles de supervivencia se registraron en ejemplares sin daño o con daño solo en los bordes; en aquellos con roturas o perforaciones en la valva el porcentaje fue cercano al 50\%. Se observó que la supervivencia disminuía a medida que aumentaba el tiempo de la experiencia.
\end{abstract}

Palabras clave: Zygochlamys patagonica, supervivencia, talla no comercial, descarte.

\section{SURVIVAL OF NON-COMMERCIAL SIZE PATAGONIAN SCALLOP DISCARDED BY THE FISHERY}

\begin{abstract}
The most important benthic fishery in extension and production of the Southwest Atlantic is that of Patagonian scallop (Zygochlamys patagonica). It is developed by four factory vessels with non-selective bottom trawl nets and processing plant on board. During the selection process the scallops of commercial size ( $\geq 55 \mathrm{~mm}$ total high) are retained and those that do not reach it $(<55 \mathrm{~mm} \mathrm{TH})$ are discarded back to sea. The purpose of this work was to determine the survival level of non-commercial specimens discarded to sea and the effect of the selection process on board. Non-commercial scallops coming from deck (control) and discard (treatment) were selected. They were classified according to the level of damage and placed in polypropylene bags to be anchored ( $\sim 100 \mathrm{~m}$ depth). The bags were retrieved 10 and $54 \mathrm{~d}$ after and the amount of live specimens in each one registered. The highest survival level was observed in individuals without damage or damaged only on the edges; in those with breaks or perforations in the shell the percentage rounded $50 \%$. It was observed that survival decreased as the time of the experiment increased.
\end{abstract}

Key words: Zygochlamys patagonica, survival, non-commercial size, discard. 
La sustentabilidad de una pesquería requiere que se conozcan los distintos factores que actúan sobre ésta, la mortalidad directa e indirecta producida por la pesca es uno de ellos. La asignación de cuotas de pesca, restricciones por tallas, cierres espaciales y temporales, son algunas de las medidas de manejo comúnmente utilizadas para disminuir la mortalidad por pesca directa (Montgomery 2008). La mortalidad por pesca indirecta tiene dos componentes principales; los individuos que son capturados y descartados, y los organismos que son dañados al entrar en contacto con las artes de pesca sin ser capturados (Jenkins y Brand 2001). La aplicación de estrategias de manejo orientadas a disminuir la mortalidad por pesca indirecta en poblaciones afectadas podría permitir la recuperación de las mismas (Kell y Bromley 2004).

La mortalidad producida por el descarte raramente es estimada en pesquerías específicas y representa una gran fuente de incertidumbre en las estimaciones de mortalidad por pesca total (Davis 2002). Existe gran variabilidad en la mortalidad entre las especies descartadas por las pesquerías (Kaiser y Spencer 1995; Bergmann y Moore 2001; Depestele et al. 2014; Schwartz et al. 2014), pero se ha visto que los bivalvos poseen tasas de supervivencia más altas que otras especies (Hill y Wassenberg 2000).

La pesquería bentónica más importante en extensión y producción del Atlántico Sudoccidental es la de vieira patagónica (Zygochlamys patagonica). Desde el inicio de la pesquería en 1996, la flota ha estado constituida por cuatro buques factoría que operan las $24 \mathrm{~h}$ a lo largo de todo el año. Los barcos vieireros pescan con tangones y redes de arrastre de fondo no selectivas, y están dotados de una planta procesadora. El proceso a bordo de los buques vieireros comienza con rolos selectores de tallas que separan las vieiras de talla comercial ( $\geq 55 \mathrm{~mm}$ de alto total de la valva) para ser procesadas de las vieiras no comerciales y especies no objetivo, las cuales son devueltas al mar. Escolar et al. (2014) encontraron que el proceso mecánico de separación aumenta significati- vamente el daño externo en las vieiras patagónicas descartadas. Recientemente se determinó la supervivencia de los invertebrados bentónicos de la captura incidental luego del proceso de selección (Schwartz et al. 2014), pero aún no se analizó cómo influye el daño recibido durante dicho proceso en la supervivencia de la vieira patagónica. En este trabajo se determinó, por medio de experiencias realizadas en el mar, la supervivencia de la vieira patagónica no comercial descartada, y cómo ésta se ve afectada por el daño recibido durante el proceso mecánico de selección de ejemplares.

Las muestras fueron tomadas a bordo del BP "Atlantic Surf I" durante la campaña dirigida a evaluar la biomasa de vieira patagónica de la Unidad de Manejo B en 2013 (Figura 1). La zona de estudio fue previamente caracterizada por su alta densidad de ejemplares de talla no comercial. Se realizaron tres lances con un tiempo promedio de

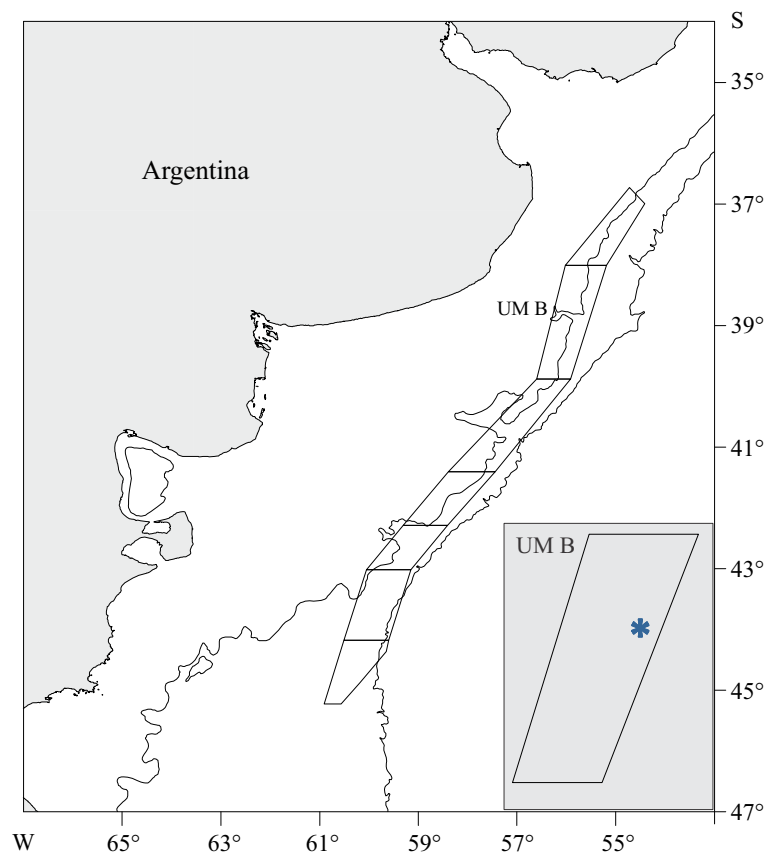

Figura 1. Unidad de Manejo B. El asterisco indica la ubicación de los lances realizados.

Figure 1. Management Unit B. The asterisk indicates the location of the trawls performed. 
13 min de arrastre a 4,5 nudos. Se utilizó el arte de pesca comúnmente empleado por el buque (red de arrastre con tangones de $22 \mathrm{~m}$ de relinga superior y mallero de $140 \mathrm{~mm}$ ).

Se seleccionaron individuos de vieira no comercial $(<55 \mathrm{~mm}$ de alto total) procedentes de la cubierta y que no pasaron por el proceso de selección (control), y del descarte (tratamiento), es decir, luego del proceso de selección a bordo (Figura 2). Algunas vieiras con talla comercial quedaron seleccionadas debido a un error durante la medición. Las vieiras seleccionadas fueron dis- puestas en seis fondeos y agrupadas de acuerdo con su nivel de daño (Tabla 1) según los criterios establecidos para moluscos por Veale et al. (2001). La selección de las vieiras comerciales en estos buques es realizada por dos tambores cribados de acero inoxidable (uno por banda), de 2,42 $\mathrm{m}$ de largo y $86,5 \mathrm{~cm}$ de diámetro, con una inclinación de $35^{\circ}$ (también conocidos como "rolos selectores").

En cada fondeo se amarraron seis grupos de aproximadamente 30 individuos cada uno, colocados en bolsas de polipropileno $(40 \times 60 \mathrm{~cm}$; malla-

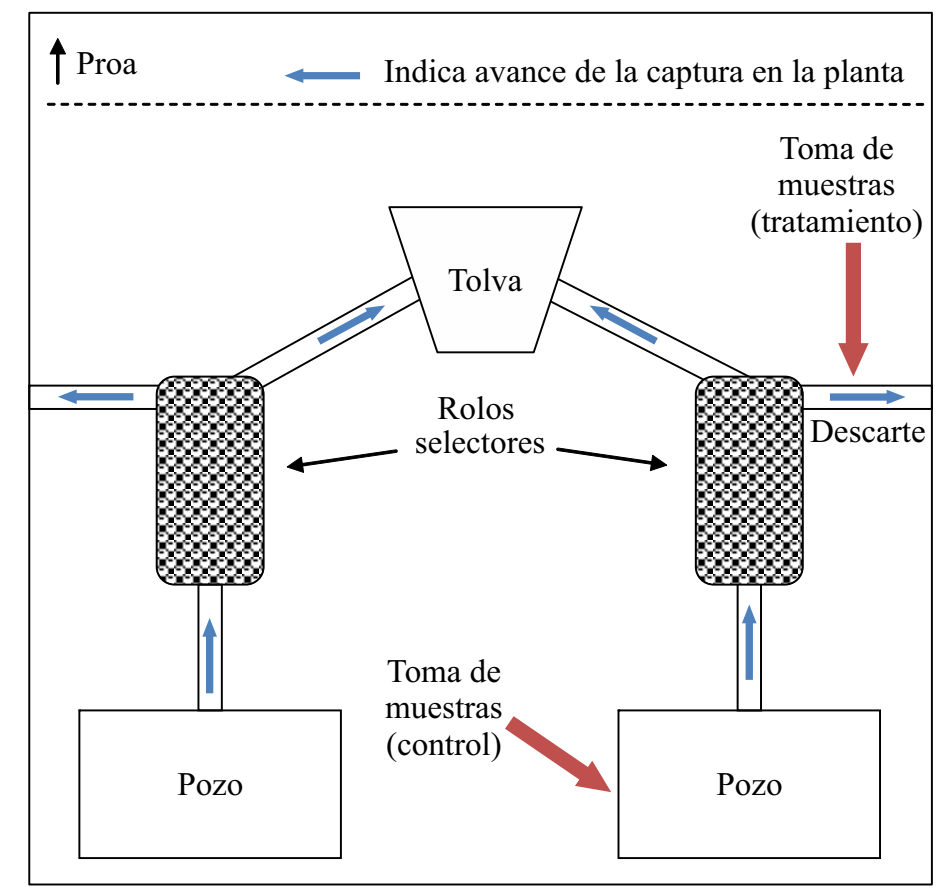

Figura 2. Esquema de selección de vieiras en la factoría del BP "Atlantic Surf I” y puntos de muestreo. Figure 2. Scheme of scallop selection in the factory of the "Atlantic Surf I" FV and sampling points.

Tabla 1. Escala de daño de la vieira patagónica (tomado de Veale et al. 2001).

Table 1. Patagonian scallop damage scale (taken from Veale et al. 2001).

\begin{tabular}{ccccc}
\hline Nivel de daño & 1 & 2 & 3 & 4 \\
\hline & Sin daño aparente & Borde de la valva dañado & Valva rota o perforada & Aplastado/muerto
\end{tabular}


do de $0,6 \mathrm{~cm}$ ) (Figura 3) quedando una réplica por daño y tratamiento en cada fondeo. Algunas de las bolsas fondeadas no llegaron a completarse con los 30 individuos debido a que no todos los niveles de daño estaban representados en cantidades suficientes en las capturas. Las bolsas se amarraron al azar a un cabo de $20 \mathrm{~mm}$ de diámetro y 200 $\mathrm{m}$ de largo, con una distancia entre bolsas de $2 \mathrm{~m}$, con boya y un muerto de $30 \mathrm{~kg}$ en el extremo para ser situada en el fondo del mar.

El tiempo de exposición al aire, tanto para el control como para el tratamiento, fue el mismo y no superó los 20 min. Para disminuir la exposición al aire, las vieiras fueron medidas (alto total) al finalizar la experiencia. De las seis líneas fondeadas tres fueron recuperadas a los $10 \mathrm{~d}$ posteriores a su fondeo (Boyas 07, 03 y 01), mientras que solo una línea fue recobrada luego de pasar $54 \mathrm{~d}$ fondeada en el mar (Boya 05). Por lo tanto, quedaron para el análisis de supervivencia cuatro réplicas por tratamiento y daño, tres con $10 \mathrm{~d}$ de fondeo y una con $54 \mathrm{~d}$. Una vez en cubierta, cada bolsa fue colocada en un balde de 101 con agua de mar, contabilizándose las vieiras vivas de acuerdo con el nivel de daño y tratamiento. Se realizó un test de ANOVA de dos factores para analizar la supervivencia entre tratamiento y niveles de daño. A posteriori se aplicó el test de Tukey de comparaciones múltiples.

Los valores de supervivencia de vieiras recuperadas a los $10 \mathrm{~d}$ de fondeados con daño de tipo 1 y 2 fueron elevados ( $~ 96 \%)$, mientras que con daño 3 fueron cercanos al 50\% (Tabla 2 A; Figura 4 A). La supervivencia entre organismos del tra-
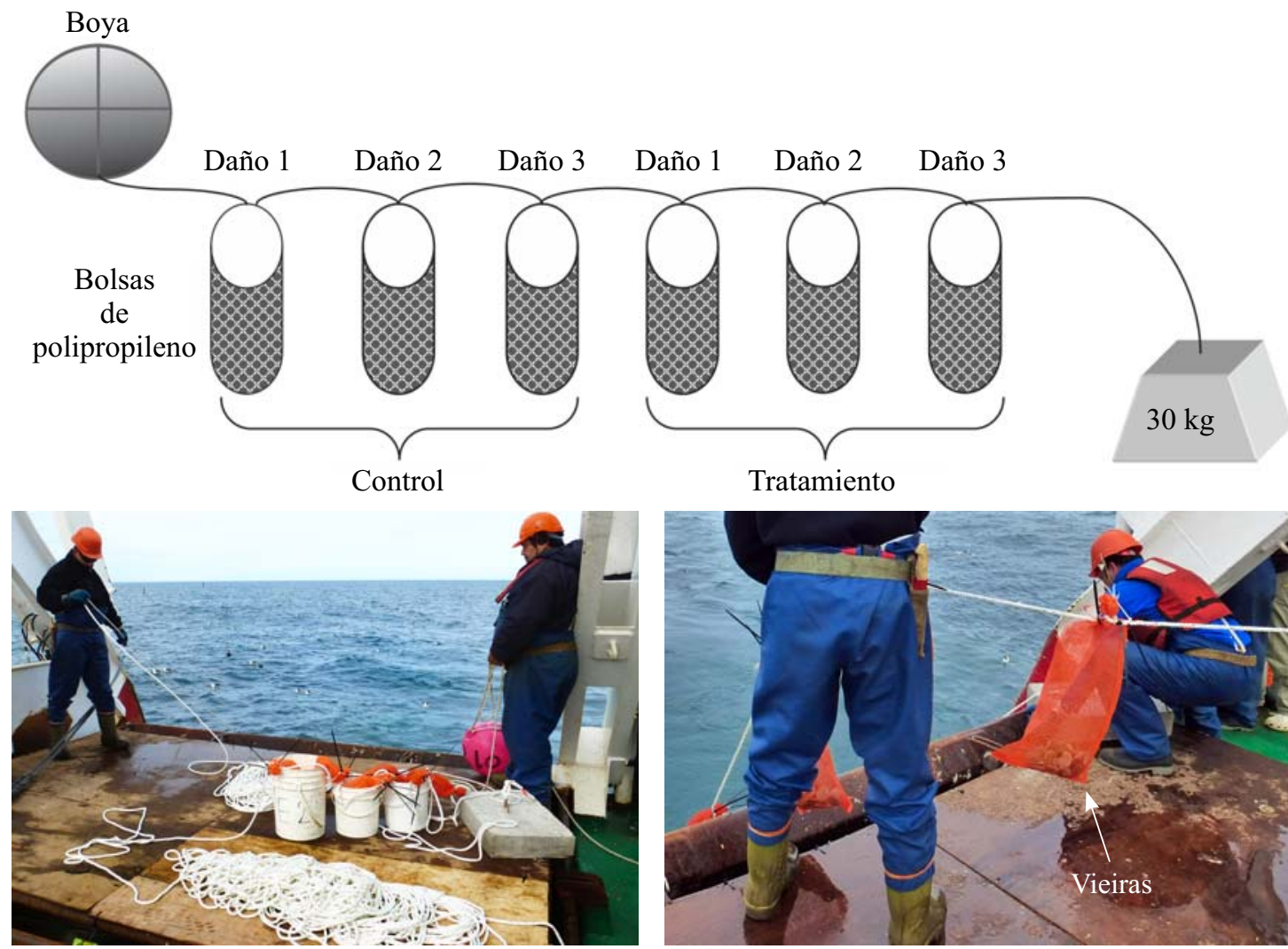

Figura 3. Línea fondeada: boya señaladora, bolsas de polipropileno con vieiras en su interior.

Figure 3. Anchored line: marker buoy, polypropylene bags with scallops inside. 
Tabla 2. Supervivencia (\%) por tratamiento y nivel de daño en individuos de vieira no comercial después de 10 (A) y 54 (B) días de fondeo.

Table 2. Survival (\%) for treatment and damage level in non-commercial scallop individuals after 10 (A) and 54 (B) days anchorage.

A

\begin{tabular}{|c|c|c|c|c|c|c|}
\hline \multirow[b]{2}{*}{ Boya } & \multicolumn{2}{|c|}{ Daño 1} & \multicolumn{2}{|c|}{ Daño 2} & \multicolumn{2}{|c|}{ Daño 3} \\
\hline & Cubierta & Descarte & Cubierta & Descarte & Cubierta & Descarte \\
\hline 7 & 83,9 & 100,0 & 100,0 & 100,0 & 24,1 & 31,3 \\
\hline 1 & 100,0 & 100,0 & 89,7 & 89,7 & 37,9 & 82,1 \\
\hline 3 & 89,7 & 100,0 & 100,0 & 100,0 & 58,6 & 36,7 \\
\hline $\mathrm{N}^{\circ}$ vivos & 81 & 85 & 82 & 86 & 35 & 44 \\
\hline $\mathrm{N}^{\circ}$ muertos & 8 & 0 & 3 & 3 & 52 & 46 \\
\hline
\end{tabular}

B

\begin{tabular}{lcccccc}
\hline & \multicolumn{2}{c}{ Daño 1 } & \multicolumn{2}{c}{ Daño 2 } & \multicolumn{2}{c}{ Daño 3 } \\
\hline Boya & Cubierta & Descarte & Cubierta & Descarte & Cubierta & Descarte \\
\hline 5 & 93,3 & 65,4 & 66,7 & 86,2 & 23,3 & 30,0 \\
$\mathrm{~N}^{\circ}$ vivos & 28 & 17 & 20 & 25 & 7 & 9 \\
$\mathrm{~N}^{\circ}$ muertos & 2 & 9 & 10 & 4 & 23 & 21 \\
\hline
\end{tabular}

A

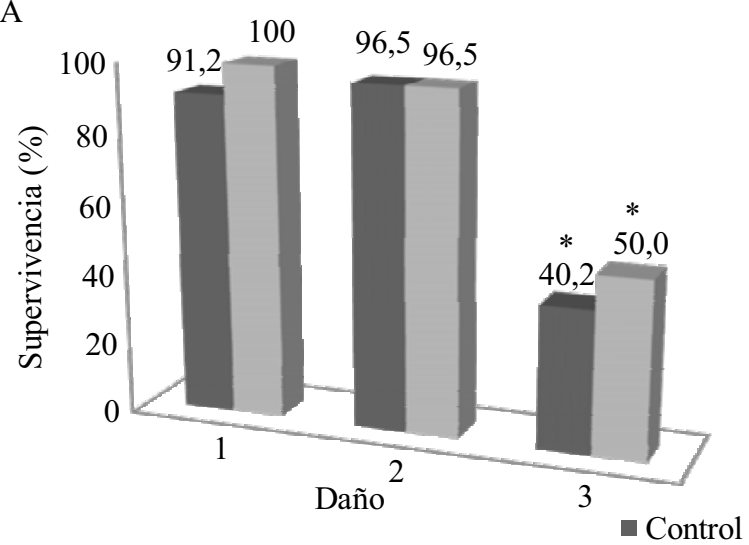

B

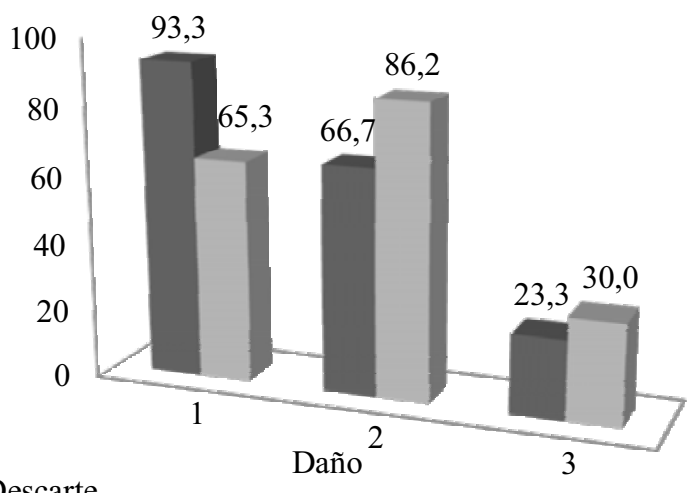

Figura 4. Supervivencia por tratamiento de vieira no comercial y nivel de daño después de 10 (A) y 54 (B) días de fondeo. *Diferencias significativas de nivel de daño.

Figure 4. Survival for treatment of non-commercial scallop and level of damage after 10 (A) and 54 (B) days anchorage. *Significant differences of level of damage. 
tamiento (descarte) no difirió significativamente a la que presentaron los organismos del control (cubierta) (Tabla 2 A; Figura 4). Solo se observaron diferencias significativas entre los niveles de daño con que fueron devueltas las vieiras al mar, tanto en el control como en el tratamiento (Tabla $3)$. Las vieiras devueltas al mar con daño 3 tuvieron supervivencia significativamente menor que aquellas con daño 1 y 2 (test de Tukey, $\mathrm{p}<0,001$ ). En el caso de la línea recuperada después de $54 \mathrm{~d}$, las vieiras con daño 1 y 2 también registraron valores de supervivencia más altos que las de daño 3 (Tabla 2 B; Figura 4 B). Por otro lado, se observó una disminución en la supervivencia de las vieiras $(\sim 22 \%)$ con el aumento del tiempo de permanencia en el fondo del mar (Figura 4). Al final del experimento, el rango de tallas de las vieiras utilizadas en el control osciló entre 11 y 60 $\mathrm{mm}(44,25 \pm 5,76)$ y en el tratamiento entre $8 \mathrm{y}$ $61 \mathrm{~mm}(48,57 \pm 6,36)$ (Figura 5 A). El porcentaje de individuos no comerciales en las muestras de cubierta y descarte fue de 96,6 y $81,1 \%$, respectivamente. Los organismos que no sobrevivieron presentaron una distribución de tallas similar a la de los sobrevivientes tanto en cubierta $(\mathrm{t}=-0,019$; $p>0,19)$ como en descarte $(t=-0,98 ; p>0,98)$ (Figura 5 B).

La supervivencia de Z. patagonica fue anteriormente estimada por Bremec et al. (2004), quienes encontraron una alta tasa (cercana al 96\%) en vieiras comerciales y no comerciales descartadas al mar. Valero y Lasta (2008) reajustaron los valores de Bremec et al. (2004) utilizando solo los individuos no comerciales y encontraron que el proceso de selección de tallas aumentó hasta 20 veces la tasa de mortalidad. En este trabajo se analizó la supervivencia de vieiras no comerciales que son las que la pesquería normalmente descarta al mar y cómo el proceso de selección afecta su supervivencia, teniéndose en cuenta el daño con el cual los individuos fueron devueltos al mar, variable que no se analizó por Bremec et al. (2004) ni Valero y Lasta (2008). Por otro lado, encontramos también altos porcentajes de supervivencia en individuos sin daño, independientemente de estar afectados o no por el proceso de selección. Los individuos que presentaron valvas rotas o perforadas tuvieron una supervivencia hasta cuatro veces menor que aquellos sanos o con daño solo sobre el margen de la valva. Los presentes resultados demuestran que daños severos en la valva afectan significativamente la supervivencia de las vieiras. Sólo se encontró una disminución en la supervivencia en individuos que presentaron el mayor nivel de daño producido por el arrastre o por el proceso de selección a bordo, contrastando lo obtenido por Valero y Lasta (2008).

Cabe aclarar que la asignación del nivel de daño estuvo basada únicamente en las características del daño externo; el daño interno y el estrés concomitantes también contribuyen a disminuir la supervivencia de los organismos que son devueltos al mar (Crowder y Murawski 1998). Asimismo, diversos estudios mostraron que las perturba-

Tabla 3. Test de ANOVA con los valores de supervivencia (\%) de vieira no comercial. GL: grados de libertad, MC: media cuadrática, F: estadístico, P: probabilidad.

Table 3. ANOVA test with non-commercial scallop survival (\%) values. GL: degrees of freedom, MC: quadratic mean, F: statistic, $P$ : probability.

\begin{tabular}{lrrrl}
\hline Factor & GL & MC & F & P \\
\hline Tratamiento & 1 & 173 & 0,85 & 0,3740 \\
Daño & 2 & 5.193 & 25,53 & $0,0001^{*}$ \\
Interacción & 2 & 44 & 0,21 & 0,8100 \\
\hline
\end{tabular}



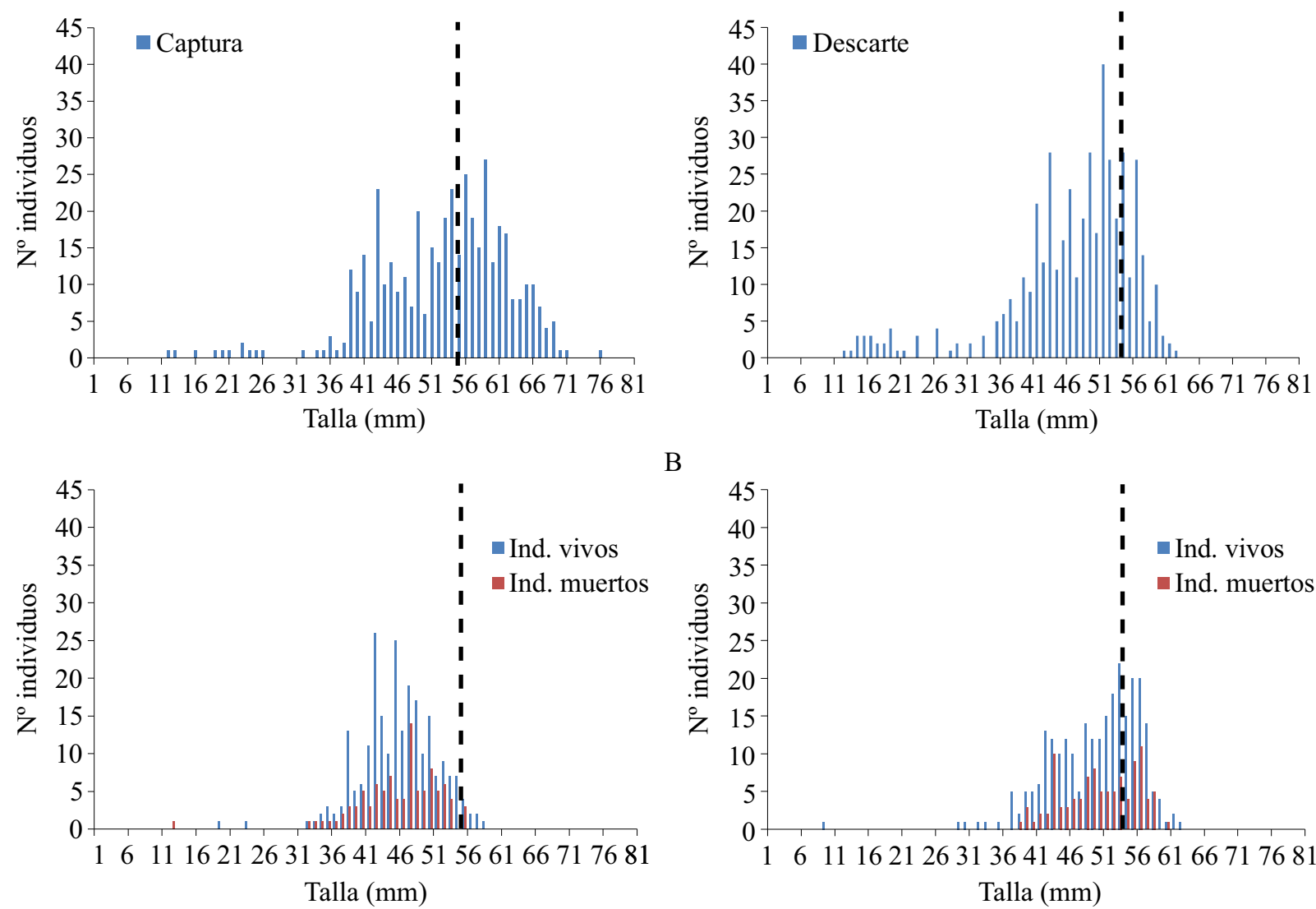

$\mathrm{B}$

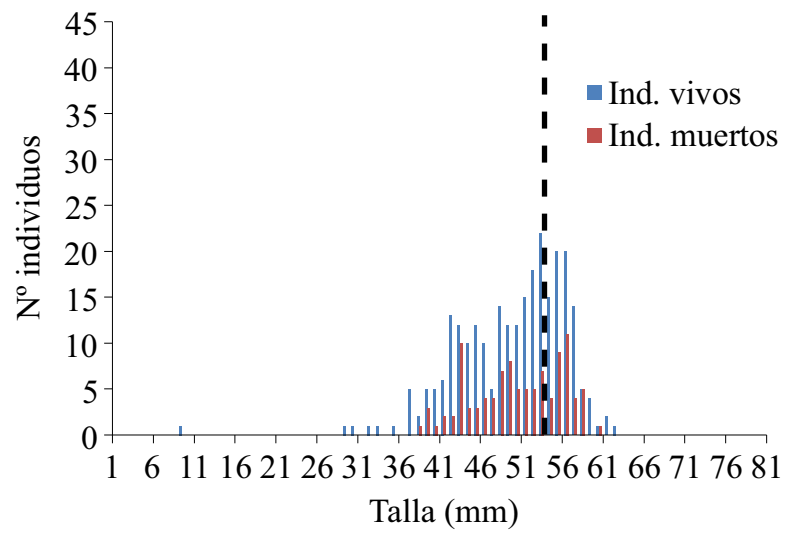

Figura 5. A) Frecuencia de tallas de la experiencia. B) Frecuencia de tallas de los individuos vivos y muertos recuperados. La línea punteada separa las vieiras no comerciales (izquierda) de las comerciales (derecha).

Figure 5. A) Size frequency of the experiment. B) Size frequency of the living and dead individuals recovered. The dotted line separates the non-commercial (left) from the commercial (right) scallops.

ciones que sufren las vieiras por estrés, exposición al aire y daños físicos, tienen un efecto negativo en la respuesta al escape de los predadores (Gruffydd 1972; Jenkins et al. 2001; Maguire et al. 2001) y aumenta su vulnerabilidad al ataque de patógenos (La Coste et al. 2001; De Zwaan et al. 2002), llevando a una disminución en la supervivencia de los ejemplares descartados.

El tiempo que los individuos permanecen en el fondo del mar durante el desarrollo de experiencias de supervivencia es fundamental, siendo que una corta duración de las mismas produciría sobreestimaciones de la supervivencia, mientras que experiencias muy largas pueden generar subestimaciones. Valero y Lasta (2008) sugieren que la duración de las experiencias de este tipo para la vieira patagónica no debería ser menor a los $12 \mathrm{~d}$, ya que observaron que el proceso de selección produce un efecto retrasado en la mortalidad. El diseño de esta experiencia contempló esta problemática, ya que la última línea fue recuperada 54 d después de su fondeo, observándose que efectivamente la supervivencia de las vieiras dañadas disminuyó con el aumento de la duración de la experiencia. Por otro lado, el uso de bolsas en lugar de linternas (dispositivo utilizado en cultivos de bivalvos) pudo generar daños en las partes blandas de las vieiras por inserción 
valvar de otros individuos apilados, produciendo una sobrestimación en la supervivencia.

Dado que se utilizaron bolsas a fin de excluir la incidencia de la predación sobre la mortalidad, las tasas de supervivencia estimadas en este trabajo podrían estar sobreestimadas (Veale et al. 2000; Maguire et al. 2002). Las vieiras que murieron durante la experiencia no presentaron una tendencia general con respecto a las tallas. Por lo tanto, la supervivencia de las vieiras descartadas no tiene relación con la talla de las mismas. Se recomienda repetir esta experiencia haciendo hincapié en individuos de talla menor a los $35 \mathrm{~mm}$ AT, los cuales no estuvieron bien representados en este trabajo.

La supervivencia estimada en este trabajo puede ser considerada representativa para la pesca comercial, ya que la misma fue determinada utilizando un arte de pesca, tiempo de arrastre, exposición al aire y proceso de selección similar a los utilizados en la pesca comercial de vieira patagónica. La supervivencia de las vieiras descartadas no está relacionada con la talla: los daños que producen los sistemas de selección no tienen relación con la talla corporal de los organismos sino que varía en función de las especies (Escolar et al. 2014). Las vieiras con daño 1 y 2 del control y tratamiento tuvieron una supervivencia alta, lo cual demuestra que el proceso de selección en sí no disminuye la supervivencia, siempre que no le genere un daño nivel 3.

Evitar que las vieiras lleguen a alcanzar un nivel de daño 3 aumentaría significativamente la supervivencia de los individuos descartados. Cambios en la estructura y funcionamiento de los sistemas de selección de tallas, como flujo de agua y revoluciones del tambor cribado, podrían analizarse en función de disminuir el daño de los animales. La devolución de individuos vivos no comerciales descartados en la pesquería de vieira patagónica aumenta la capacidad de sustentabilidad del recurso, la cual podría verse afectada por daños producidos sobre los organismos durante el descarte de los mismos.

Contribución INIDEP Nº 2194.

\section{REFERENCIAS}

Bergmann M, Moore PG. 2001. Mortality of Asterias rubens and Ophiura ophiura discarded in the Nephrops fishery of the Clyde Sea area, Scotland. ICES J Mar Sci. 58: 531-542.

Bremec C, Lasta M, Hernández D. 2004. Survival of Patagonian scallop (Zygochlamys patagonica, King and Broderip, 1832) after the size selection process on commercial fishing vessels. Fish Res. 66: 49-52.

Crowder L, Murawski S. 1998. Fisheries bycatch: implications for management. Fisheries. 23: 8-15.

DAVIS M. 2002. Key principles for understanding fish by-catch discard mortality. Can J Fish Aquat Sci. 59: 1834-1843.

Depestele J, Desender M, Benoît HP, Polet H, VINCX M. 2014. Short-term survival of discarded target fish and non-target invertebrate species in the "eurocutter" beam trawl fishery of the southern North Sea. Fish Res. 154: 82-92.

De ZwaAn A, Babarro J, Monari M, Cattani O. 2002. Anoxic survival potential of bivalves: (arte) facts. Comp. Biochem. Physiol A. 131: 615-624.

Escolar M, Schwartz M, Marecos A, Herrera S, Diaz R, Schejter L, CAmpodónico M, BREMEC C. 2014. Estimación del daño ocasionado a los invertebrados bentónicos por el proceso de selección a bordo en buques de la flota comercial de vieira patagónica Inf Invest INIDEP No 73/2014. 45 p.

GRUFFYDD L. 1972. Mortality of scallops on a Manx scallop bed due to fishing. J Mar Biol Assoc UK. 52: 449-454.

Hill B, Wassenberg T. 2000. The probable fate of discards from prawn trawlers fishing near coral reefs: a study in the northern Great Barrier Reef, Australia. Fish Res. 48: 277-286.

Jenkins S, Beukers-Stewart B, Brand A. 2001. Impact of scallop dredging on benthic 
megafauna: a comparison of damage levels in captured and non-captured organisms. Mar Ecol Prog Ser. 215: 297-301.

Jenkins S, BRAnD A. 2001. The effect of dredge capture on the escape response of the great scallop, Pecten maximus (L.): implications for the survival of undersized discards. J Exp Mar Biol Ecol. 266: 33-50.

Kaiser M, Spencer B. 1995. Survival of by-catch from a beam trawl. Mar Ecol Prog Ser. 126: 31-38.

Kell L, Bromley P. 2004. Implications for current management advice for North Sea plaice (Pleuronectes platessa L.): Part II. Increased biological realism in recruitment, growth, density-dependent sexual maturation and the impact of sexual dimorphism and fishery discards. J Sea Res. 51: 301-312.

la Coste A, Jalabert F, Malham S, Cueff A, Poulet S. 2001. Stress and stress-induced neuroendocrine changes increase the susceptibility of juvenile oysters (Crassostrea gigas) to Vibrio spendidus. Appl Environ Microbiol. 67: 2304-2309.

Maguire J, Coleman A, Jenkins S, Burnel G. 2001. Effects of dredging on undersized scallops. Fish Res. 56: 155-165.

Maguire J, Jenkins S, Burnell G. 2002. The effects of repeated dredging and speed of tow on undersized scallops. Fish Res. 58: 367-377.
Montgomery J. 2008. Discarding in the Isle of Man Queen Scallop, Aequipecten opercularis, fishery. The effect of on-deck sorting processes upon survival potential of undersized queen scallops [MSc]. Bangor University. $61 \mathrm{p}$.

Schwartz M, Escolar M, Marecos A, CAmpoDÓNICO S. 2014. Supervivencia de invertebrados bentónicos en la pesquería de vieira patagónica. Inf Invest INIDEP No 51/2014. 11 p.

VALERO J, LASTA M. 2008. Estimating survival of discarded scallops in the Patagonian scallop fishery: comment on "Survival of Patagonian scallop (Zygochlamys patagonica, King and Broderip, 1832) after the size selection process on commercial fishing vessels", by Bremec et al. 2004. Fish Res. 90: 313-315.

Veale L, Hill A, Brand A. 2000. An in situ study of predator aggregations on scallop (Pecten maximus (L)), dredge discards using a static time-lapse camera system. J Exp Mar Biol Ecol. 255: 111-129.

Veale L, Hill A, Hawkins S, Brand A. 2001. Distribution and damage to the by-catch assemblages of the northern Irish Sea scallop dredge fisheries. J Mar Biol Assoc UK. 81: $85-96$.

Recibido: 13-05-2015

Aceptado: 01-08-2018 
